\title{
Review: limited evidence exists on the effect of psychological coping styles on cancer survival or recurrence
}

\author{
Petticrew M, Bell R, Hunter D. Influence of psychological coping on survival and recurrence in people with cancer: \\ systematic review. BMJ 2002;325:1066-9.
}

\section{QUESTION: In patients with cancer, do psychological coping styles such as fighting spirit, helplessness/hopelessness, denial, and avoidance improve survival or prevent recurrence?}

\section{Data sources}

Studies up to June 2002 were identified by searching Medline, PsycINFO, ASSIA, EMBASE/Excerpta Medica, Cancerlit, CINAHL, and Dissertation Abstracts. Bibliographies of relevant articles were reviewed, and experts were contacted for unpublished information.

\section{Study selection}

Studies published in any language were selected if they were cohort studies, patients had cancer, and outcomes included mortality, survival, or recurrence. Exclusion criteria included studies of the association between coping and immune responses or other biochemical markers (if this was the only outcome reported) and studies of personality types (eg, "type C" personality).

\section{Data extraction}

Data were extracted on sample size, styles of coping, site of cancer, duration of follow up, study quality (adjustment for potential confounding factors, use of an inception cohort, and assessment of coping early in the disease process), and outcomes.

\section{Main results}

37 studies met the selection criteria. 26 studies assessed

Source of funding: The Scottish Executive Department of Health.

For correspondence: Dr M Petticrew, MRC Social and Public Health Sciences Unit, Glasgow, UK. mark@msoc.mrc.gla.ac.uk helplessness/hopelessness, stoic acceptance and fatalism, anxious and depressive coping, problem focused coping, and emotional focused coping. The results are summarised in the table.

\section{Conclusion}

In patients with cancer, evidence on the association between psychological coping styles (eg, fighting spirit, helplessness/hopelessness, denial, and avoidance) and survival from or recurrence of cancer is inconclusive.

\section{COMMENTARY}

Patients with cancer are often encouraged by family members and healthcare providers to "think positively" or use optimistic coping strategies to augment the effectiveness of treatment. The study by Petticrew et al provides an important summary of the available evidence regarding the effect of psychological coping on cancer survival and recurrence.

Strengths of this study include the comprehensive search of data sources, relevant criteria for inclusion and exclusion of studies, and data extraction methods. However, the use of terms in the psychological literature can be inconsistent. Providing a clear definition of psychological coping and reporting the key terms used in the search strategy would increase reader confidence that all relevant studies had been identified. Studies were also analysed according to types of coping styles. How studies were categorised is unclear, which limits the reader's ability to validate the interpretation of individual study results.

However, it is unlikely that these methodological issues substantially influenced the inconclusive study results. An important message from this review is the need for better research involving methodologically rigorous studies with large sample sizes, greater consistency in the use of methods to evaluate coping styles, and longer follow up. The review also focused on studies that evaluated coping near the time of diagnosis. The inconsistent results may reflect the tendency for people to use a variety of coping strategies that vary with time and perceptions of stressful events. ${ }^{1}$

The results of this study are relevant to oncology and palliative care nurses. Patients with cancer should not be pressured to adopt a particular coping style or use specific coping strategies in an effort to improve their chances for survival or prevent a recurrence of their cancer. Nurses can also help relieve patients from the burden or responsibility they may feel to "cope better" in order to improve treatment outcomes.

Denise Bryant-Lukosius, RN, MScN Assistant Professor McMaster University Advanced Practice Nurse Hamilton Regional Cancer Centre Hamilton, Ontario, Canada

1 Dunkel-Schetter C, Feinstein LG, Taylor SE, et al. Patterns of coping with cancer. Health Psychol 1992;11:79-87. 